\title{
Simulation of free-electron lasers seeded with broadband radiation
}

\author{
S. I. Bajlekov, ${ }^{1, *}$ W. M. Fawley, ${ }^{2,3}$ C. B. Schroeder, ${ }^{2}$ R. Bartolini,,${ }^{4,5}$ and S. M. Hooker ${ }^{1}$ \\ ${ }^{1}$ Department of Physics, University of Oxford, OX1 3PU, United Kingdom \\ ${ }^{2}$ Lawrence Berkeley National Laboratory, University of California, Berkeley, California 94720, USA \\ ${ }^{3}$ Sincrotrone Trieste, Trieste, Italy \\ ${ }^{4}$ Diamond Light Source Ltd., Oxfordshire, OX11 OQX, United Kingdom \\ ${ }^{5}$ John Adams Institute, University of Oxford, OX1 3RH, United Kingdom
}

(Received 10 March 2011; published 27 June 2011)

\begin{abstract}
The longitudinal coherence of free-electron laser (FEL) radiation can be enhanced by seeding the FEL with high harmonics of an optical laser pulse. The radiation produced by high-harmonic generation (HHG), however, has a fast-varying temporal profile that can violate the slowly varying envelope approximation and limited frequency window that is employed in conventional free-electron laser simulation codes. Here we investigate the implications of violating this approximation on the accuracy of simulations. On the basis of both analytical considerations and 1D numerical studies, it is concluded that, for most realistic scenarios, conventional FEL codes are capable of accurately simulating the FEL process even when the seed radiation violates the slowly varying envelope approximation. We additionally discuss the significance of filtering the harmonic content of broadband HHG seeds.
\end{abstract}

DOI: 10.1103/PhysRevSTAB.14.060711

PACS numbers: 41.60.Cr

\section{INTRODUCTION}

The ability of free-electron lasers to produce coherent, high-power pulses of tunable radiation in spectral regions not accessible with conventional sources makes them highly attractive to many areas of research $[1,2]$. The recent advances at extreme ultraviolet and hard $\mathrm{x}$-ray wavelengths at the Linac Coherent Light Source [3], FLASH [4], and SPring-8 (Japan) [5] have opened a new era of research for the light source user community.

Radiation in very short-wavelength free-electron lasers (FELs) has generally been produced through the process of self-amplification of spontaneous emission (SASE) [6] where random microbunching (shot noise) in the driving electron bunch provides the initial seed for the FEL instability, which is then strongly amplified through an undulator typically many gain lengths long. The longitudinal coherence of SASE output is normally limited to the so-called cooperation length [7], $L_{c}=\left(\lambda_{R} / \lambda_{u}\right) L_{G}$, where $\lambda_{R}$ is the FEL-resonant wavelength, $\lambda_{u}$ is the undulator period, and $L_{G}$ is the gain length.

The longitudinal coherence length of FEL radiation can be extended beyond $L_{c}$ via seeding by an appropriately coherent external radiation source. However, conventional lasers operating at wavelengths shorter than $\sim 200 \mathrm{~nm}$ wavelength are not readily available. Recently, there has been great interest at numerous laboratories in seeding FELs directly with coherent radiation produced by

\footnotetext{
*s.bajlekov1@physics.ox.ac.uk.
}

Published by the American Physical Society under the terms of the Creative Commons Attribution 3.0 License. Further distribution of this work must maintain attribution to the author(s) and the published article's title, journal citation, and DOI. high-harmonic generation (HHG) [8] from intense visible laser pulses interacting with a gas. This scheme has been tested experimentally in single pass FEL amplifiers to wavelengths as short as $\sim 60 \mathrm{~nm}$ (13th harmonic of Ti: sapphire) $[9,10]$ and, in principle, can work down to the few-nm regime.

In $\mathrm{HHG}$ an intense drive laser pulse ionizes atoms in a low-density gas medium. Each ionized electron is accelerated in the continuum by the laser's electric field and can recollide with the parent ion [8]. Upon recombination, the energy gained by the electron due to the laser field is emitted in the form of a high-energy photon. The most energetic photons are emitted by electrons ionized at a fixed offset from the extrema of the driving pulse. Because of this condition, HHG emission nominally comprises a series of subfemtosecond spikes, one for each half cycle of the driving laser. This corresponds to a spectrum containing all odd harmonics of the driving frequency, up to a cutoff determined by the wavelength and intensity of the driving laser, as well as the ionization potential of the gas. One characteristic of HHG sources is that the power at low harmonics (e.g., $q \leq 7$ ) of the drive laser is many orders of magnitude greater than those at high harmonics (e.g., $q \geq 21$ ).

The effects of an HHG seed's rich spectral content and subfemtosecond temporal structure upon FEL gain have been considered previously by Giannessi et al. [11], McNeil et al. [12], and Wu et al. [13], among others. In Refs. [11,12] the authors dismiss such effects reasoning that, since the FEL gain bandwidth typically encompasses only a single harmonic of the seed, the presence of the other harmonics will therefore negligibly affect FEL dynamics. In both cases, simple filtering of the HHG seed in Fourier space was carried out in order to use numerical 
codes based on the slowly varying envelope approximation (SVEA); in general, such codes support only a limited frequency domain around a central frequency. Such filtering can lead to noncausal changes in the temporal structure, e.g., making radiation artificially appear in the valleys between the strong HHG spikes. Wu et al. discuss smearing of the seed's temporal structure within an analytical framework.

Here we revisit the issue of the use of SVEA in FEL simulation and, more specifically, its use when modeling the HHG seed's temporal and spectral structure vis-à-vis FEL amplification. Since the SVEA assumes a radiation amplitude and phase that implicitly varies slowly compared to a central wavelength $\lambda_{0}$ and a radiation spectral content that can be mapped to a relatively narrow region around $\lambda_{0}$, it is not immediately clear that conventional FEL codes operating within the constraints of SVEA will accurately capture all the important details of HHG seeding. For instance, high-harmonic generation can produce bursts of radiation as short as $\approx 100$ attoseconds [14], which for a resonant FEL wavelength $\lambda_{R}=32 \mathrm{~nm}$ corresponds to just a single cycle and thus contains a broad spectral content extending far beyond the $32-\mathrm{nm}$ region.

A related approximation used by nearly all conventional FEL simulation codes is so-called undulator-period averaging in which the particle-field interaction is locally in time and $z$ reduced to only the resonant component. Since a particle slips relative to the radiation field one resonant wavelength in time for each undulator period traveled in $z$, as with SVEA it is not clear that such averaging will work well for temporal radiation structures with spikes of order a single resonant period in duration. At the other extremewavelengths much longer than that corresponding to FEL resonance-it is not clear that the strong radiation components in the HHG spectrum at the low harmonics of the drive laser can be safely neglected in terms of their effects upon the particle dynamics and, if not, whether the combination of spectral filtering and undulator-period averaging accurately capture these effects.

In the remainder of this paper we study these various issues beginning with Sec. II where we give qualitative arguments for why the SVEA in 1D geometries can handle a surprisingly wide spectral content range for an FEL seed. In Sec. III we introduce the non-SVEA code AURORA and discuss our procedures for filtering the full spectral content of a wideband seed for use in a conventional, SVEA-based code similar to PERSEO [15]. We also summarize a linearized Vlasov-Maxwell model whose details are given in the Appendix that is also used for later comparison of results. We then use these tools in Sec. IV to explore non-SVEA effects in the FEL response to first extremely narrow radiation spikes and then to the rich spectrum of an HHG seed, discussing the regimes in which an HHG's seed's spectral content, especially the high intensity, low harmonic components, may affect FEL gain at the resonant component. Here we also examine the case where the gain bandpass is sufficiently broad to include multiple HHG harmonics. We conclude in Sec. V with a brief summary.

\section{THE SLOWLY VARYING ENVELOPE APPROXIMATION IN FEL CODES}

Here we examine the validity of the SVEA in the context of seeded FELs, where the input radiation's longitudinal profile violates the SVEA.

Simulating gain in a free-electron laser involves the evaluation of the combined action of the undulator and radiation fields on the electrons at each position along the undulator, as well as the evaluation of the emitted field, based on the electron positions and velocities at that point. Generally these interactions are averaged over one or more undulator periods, with all variables propagated from one undulator position to another. At any given interaction point within the classical framework, the effect of the radiation on the electrons is independent of the emission of radiation by the electrons. From a numerical standpoint, the effect of a seed is entirely mediated by the electrons, in the sense that the seed causes energy modulation and then microbunching, which in turn causes emission of radiation. When the microbunched electrons radiate, the presence of the seed radiation has no explicit effect on the newly emitted radiation and the two radiation fields add linearly. This view of the emission process is completely independent of the adoption of the SVEA.

Radiation emission is calculated from Maxwell's wave equation for the transverse components of the field's magnetic vector potential in the Lorenz gauge, $\vec{A}$, and for the source current density, $\vec{J}$,

$$
\left(\frac{\partial^{2}}{\partial z^{2}}-\frac{1}{c^{2}} \frac{\partial^{2}}{\partial t^{2}}\right) A_{\perp}=-\frac{1}{\epsilon_{0} c^{2}} J_{\perp},
$$

where $c$ is the speed of light and $\epsilon_{0}$ is the vacuum permittivity. In this paper we restrict ourselves to a 1D model and have accordingly dropped the diffraction term, $\nabla_{\perp}^{2} A_{\perp}$.

At this point, one may proceed in two different fashions in regards to the eventual applicability of the SVEA in the context of FEL emission. If we exploit our 1D assumption without worrying about a future extension to higher dimensionality, one may factor the left-hand side of Eq. (1) to obtain

$$
\left(\frac{\partial}{\partial z}+\frac{1}{c} \frac{\partial}{\partial t}\right)\left(\frac{\partial}{\partial z}-\frac{1}{c} \frac{\partial}{\partial t}\right) A_{\perp}=-\frac{1}{\epsilon_{0} c^{2}} J_{\perp} .
$$

With $B_{\perp}=\nabla \times A_{\perp}$ and $E_{\perp}=-\partial A_{\perp} / \partial t$, one may separate Eq. (2) into two separate equations for the forwardgoing wave $E_{\perp}-\vec{z} \times c B_{\perp}$ and the backward-going wave $E_{\perp}+\vec{z} \times c B_{\perp}$. In the FEL context, this result was previously obtained by Maroli [16]; in the plasma simulation field this method of advancing Maxwell's equation for electromagnetic codes has been known since at least the 
1970s [17] (and perhaps dates back 50 years earlier to Sommerfeld). Given our focus upon short-wavelength FELs for which there is no resonant gain in the backward wave, we neglect it and set $c B_{\perp}=\vec{z} \times E_{\perp}$ exactly. We then make a Galilean transformation to the frame of reference moving forward in $z$ at velocity $c$ by carrying out the substitutions $\hat{t}=t-z / c$ and $\hat{z}=z$, resulting in the exact first order equation

$$
\frac{\partial}{\partial \hat{z}} E_{\perp}(\hat{t})=-\frac{1}{2 \epsilon_{0} c} J_{\perp} .
$$

This equation can be integrated forward in $z$ in either the time or frequency domain without requiring adoption of the SVEA (of course, accurate evaluation of highfrequency components necessitates that $E_{\perp}$ and $J_{\perp}$ be resolved sufficiently finely on the numerical $\hat{t}$ grid).

However, extension to higher dimensionality and including physical effects such as diffraction brings in nontrivial complications, even for the forward-going radiation wave because in the off-axis directions one will no longer have $\vec{E}$ and $\vec{B}$ lying entirely within the transverse plane. Accordingly, we proceed along a second path that will illustrate the approximations required for the SVEA and, using the substitutions mentioned above, rewrite Eq. (1) as

$$
\left(\frac{\partial^{2}}{\partial \hat{z}^{2}}-\frac{2}{c} \frac{\partial^{2}}{\partial \hat{z} \partial \hat{t}}\right) A_{\perp}=-\frac{1}{\epsilon_{0} c^{2}} J_{\perp} .
$$

The field and source current may be represented as the products of envelopes and oscillations at an angular frequency $\omega_{0}=2 \pi c / \lambda_{0}$, where $\lambda_{0}$ is typically chosen equal to the resonant wavelength $\lambda_{R}$ (and in an SVEA code $\omega_{0}$ is the center of the frequency window),

$$
A(\hat{z}, \hat{t})=A_{0}(\hat{z}, \hat{t}) e^{-i \omega_{0} \hat{t}}, \quad J(\hat{z}, \hat{t})=J_{0}(\hat{z}, \hat{t}) e^{-i \omega_{0} \hat{t}},
$$

where we have dropped the $\perp$ subscript for clarity. Notably, this representation makes no assumptions regarding the envelopes-it still allows for variations on arbitrarily short time scales. Hereby Eq. (4) can be recast as

$$
\frac{2 i \omega_{0}}{c} \frac{\partial A_{0}}{\partial \hat{z}}=-\frac{1}{\epsilon_{0} c^{2}} J_{0}-\frac{\partial^{2} A_{0}}{\partial \hat{z}^{2}}+\frac{2}{c} \frac{\partial^{2} A_{0}}{\partial \hat{z} \partial \hat{t}} .
$$

The slowly varying envelope approximation consists of dropping the last two terms on the right-hand side (rhs) of Eq. (5). SVEA is applicable provided that (i) $\left|\partial^{2} A_{0} / \partial \hat{z}^{2}\right| \ll 2 \omega_{0} / c\left|\partial A_{0} / \partial \hat{z}\right|$ and (ii) $\left|\partial^{2} A_{0} / \partial \hat{z} \partial \hat{t}\right| \ll$ $2 \omega_{0}\left|\partial A_{0} / \partial \hat{z}\right|$. Condition (i) applies strongly for the forward wave component of essentially all realistic FEL configurations, especially those with $\lambda_{R} \ll \lambda_{u}$ since for $\rho \leq 10^{-2}$ the exponential gain length $L_{G} \geq 5 \lambda_{u} \gg \lambda_{R}$, and the rate of change of $A_{0}$ along the undulator scales as the gain length, $\partial A_{0} / \partial \hat{z} \sim A_{0} / L_{G}$. Here $\rho$ is the FEL Pierce parameter, $\quad \rho=1 /\left(2 \gamma_{R}\right)\left[\left(I / I_{A}\right)\left(\lambda_{u} a_{u}[J J]\right)^{2} /\left(2 \pi \Sigma_{b}\right)\right]^{1 / 3}$, where $\gamma_{R}$ is the electron bunch resonant Lorentz factor, $I / I_{A}$ is the bunch peak current normalized to the Alfvén current, $\Sigma_{b}$ is the bunch cross section, $\lambda_{u}$ and $a_{u}$ are respectively the undulator period and rms strength parameter, and for a planar undulator $[\mathrm{JJ}]$ is the difference of Bessel functions $\left[J_{0}(\xi)-J_{1}(\xi)\right]$ with $\xi \equiv a_{u}^{2} /\left[2\left(1+a_{u}^{2}\right)\right]$. The gain length is $L_{G}=\lambda_{u} /(4 \sqrt{3} \pi \rho)$. Although the backward wave can violate condition (i) as mentioned above, there is no resonant gain for this component and its effective strength is a factor $\sim O\left(\gamma^{2} \lambda_{u} / l_{b}\right)$ less than the forward wave where $l_{b}$ is the electron bunch length.

With regard to condition (ii) in Eq. (5), the $\partial^{2} A_{0} / \partial \hat{z} \partial \hat{t}$ term is a correction that is relevant only when the change in the field, $\partial A_{0} / \partial \hat{z}$, is fast varying in $\hat{t}$-therefore, the initial radiation seed, which is invariant with $z$ in the $1 \mathrm{D}$ approximation whatever its spectral content, does not contribute to this term.

Strong initial modulations on the bulk current or externally produced initial microbunching bring about effects such as coherent spontaneous emission that might violate condition (ii); these have been discussed elsewhere $[18,19]$ and are outside the scope of our focus on seeded FELs. On the other hand, whether or not such modulations in the electron beam may develop in $z$ in response to structures in the seed whose characteristic frequency $\omega$ is far from $\omega_{0}$ depends on their evolution with $z$. Realistic FELs with $\rho \ll 1$ will have resonant growth only for modulations whose frequencies lie within a narrow bandpass $\Delta \omega \sim$ $\rho \omega_{0}$ centered on $\omega_{0}$ (ignoring higher harmonics and presuming $\lambda_{0}=\lambda_{R}$ ). Nonresonant seed structures are expected to lead at most to short-lived (in $z$ ) modulations in electron beam energy and ponderomotive phase. Unless these modulations are sufficiently strong to either affect growth at the central resonant wavelength $\lambda_{R}$ (e.g., strongly increasing the coarse-grained energy spread and thus reducing gain) or to shift growth to neighboring wavelengths (e.g., sideband formation), one expects condition (ii) to remain valid and application of the SVEA to be justifiable in the context of coherent radiation emission even when the initial field envelope is fast varying (in phase or amplitude) compared to $\omega_{0}$. In the next section we describe two 1D codes we used to explore these expectations numerically: a non-SVEA code which includes the last term on the rhs of Eq. (5), and a conventional SVEA code that does not.

\section{SIMULATION CODE, SEED FILTERING, AND ANALYTICAL VLASOV MODEL DESCRIPTIONS}

\section{A. Non-SVEA, 1D code AURORA}

The non-undulator-averaged, non-SVEA code used is AURORA [20]. As a multifrequency code it shares a number of features with MUFFIN by Piovella [19]. We note that Campbell et al. [21] have developed a fully 3D, non-SVEA code that also follows Piovella and advances the wave equation [i.e., our Eq. (5) with diffraction terms] in Fourier space. In AURORA, the FEL interaction is simulated within a moving time window of duration $L \lambda_{R} / c$, where $L$ is an integer, and adopts periodic boundary conditions. As 
the window propagates down the undulator at a constant speed of $+c$, the evolution of the radiation field and the electron positions and energies are computed at $N$ points per undulator period. The coordinate within the window is the phase measured relative to a resonant, monochromatic plane wave, $\varphi=k_{R}(z-c t)$, where $k_{R}=2 \pi / \lambda_{R}$ is the resonant wave number. The usual ponderomotive phase is $\theta=\varphi+Z$, where $Z=k_{u} z$ is the distance along the undulator normalized by the undulator wave number $k_{u}=$ $2 \pi / \lambda_{u}$

In AURORA the radiation is represented by the transversely polarized, normalized magnetic vector potential, $a(z, \varphi)=e A_{x}(z, \varphi) / m c$. Recording this on a grid of $N$ points per wavelength allows a simulation bandwidth of $2 \lambda_{R} / N<\lambda<L \lambda_{R}$. The electron bunch is represented by a set of $P$ macroparticles whose weights, $w_{i}$, determine the number of electrons represented by each. The macroparticles are free to move within the window (i.e., are not confined to "bins," as in undulator-period-averaged codes) with any electrons escaping through the back of the window reentering through the front. Initial shot-noise microbunching can either be modeled by a Poissonian distribution of either macroparticle displacement [22,23] or weights $w_{i}$ as suggested by McNeil et al. [24].

The bunch cross section $\Sigma_{b}$ is assumed to be constant and matched to the radiation cross section, and, given our 1D geometry, diffraction effects are not considered. Our studies adopt a linearly polarized undulator with constant period $\lambda_{u}$ and constant rms strength parameter $a_{u}$. The transverse current density at location $z, J_{x}(z, \varphi)$, is evaluated through

$$
J_{x}(z, \varphi)=\frac{e \sqrt{2} a_{u}}{\Sigma_{\mathrm{b}}} \cos \left(k_{u} z\right) \sum_{i=1}^{P} \frac{w_{i}}{\gamma_{i}} C\left(\varphi-\varphi_{i}\right),
$$

where $k_{u}=2 \pi / \lambda_{u}$ is the undulator wave number, $i$ runs over the macroparticle indices, $\gamma_{i}$ are the macroparticle Lorentz factors, and $\varphi_{i}$ are the resonant radiation phases at the macroparticle locations. The distribution $C$ represents the particle-in-cell weighting applied to the macroparticles in order to project their distribution onto the radiation grid. In AURORA, the current density $J_{x}$ is transformed to Fourier space, $\tilde{J}_{x}(z, \omega)=\int d t J_{x}(z, t) e^{i \omega t}$, and filtered with a Gaussian of width $\sim 30 \omega_{0}$ centered at $\omega=0$ in order to reduce high-frequency noise. The result is then inserted into the Fourier transform of Eq. (4), omitting the second derivative with respect to $z$ :

$$
\frac{d}{d z} \tilde{A}_{x}(z, \omega)=\frac{1}{2 i \omega} \frac{1}{\epsilon_{0} c} \tilde{J}_{x}(z, \omega),
$$

to compute the field evolution.

Macroparticle energies and positions are propagated using

$$
\frac{d \gamma_{i}}{d z}=-\frac{\sqrt{2} e a_{u}}{\gamma_{i} m c^{2}} \cos \left(k_{u} z\right) E_{x}\left(z, \varphi_{i}\right)
$$

and

$$
\frac{d \varphi_{i}}{d z}=-\frac{k_{R}}{2 \gamma_{i}^{2}}\left\{1+a_{u}^{2}\left[1+\cos \left(2 k_{u} z\right)\right]\right\}
$$

with $E_{x}=-\partial A_{x} / \partial t$. The cosine term in Eq. (9) corresponds to the oscillation of the electron's longitudinal velocity that is characteristic of planar undulators, naturally leading to the emission of odd radiation harmonics of the fundamental. Since AURORA does not explicitly compute the transverse particle trajectories, the above equations omit higher-order terms (relating $z_{i}$ and $\varphi_{i}$ ) in the longitudinal trajectories. As normally done in FEL simulation codes, the radiation field contribution (i.e., the quiver velocity) to Eq. (9) is neglected, and macroparticle motion is assumed to be dominated by the undulator field. We have verified that this is justified since $a \ll a_{\mathrm{u}}$ for all normalized seed and undulator potentials considered in Sec. IV.

Finally, the computation of long-scale length space-charge forces is implemented in AURORA, but was not switched on for the simulations presented here. Corresponding simulations including space-charge effects were run in order to verify that they do not introduce any new physics or otherwise alter our conclusions.

\section{B. SVEA code and the initialization of the radiation field}

The conventional undulator-period-averaged SVEA code used for the studies here is a 1D code based on the model of PERSEO [15], against which it has been benchmarked. The time grid is typically $\lambda_{0} / c$ wide; hence, there is support for angular frequencies in the range $\left[\omega_{0} / 2,3 \omega_{0} / 2\right]$. A key first step towards using the SVEA code to simulate the FEL response to a broadband seed is the requirement to rebin the seed onto a relatively coarse (relative to that of AURORA) time grid, while ensuring important characteristics of the seed are retained. The rebinning process consists of two steps: First, a Gaussian spectral filter of root-mean-square (rms) width $\omega_{0} /(2 \pi)$ centered on $\omega_{0}$ is applied. Here $\omega_{0}=2 \pi c / \lambda_{0}$, where $\lambda_{0}$ is the bin size, which we assume to be equal to the FEL's resonant wavelength $\lambda_{R}$. This spectral filter serves to remove spectral components of extremely high and extremely low frequencies that may otherwise lead to aliasing anomalies; at the same time the filter remains broad enough to preserve the temporal profile of the seed - and therefore of the FEL output during early stages of gain-on scales of $\gtrsim \lambda_{0}$.

Second, the filtered radiation field is transformed to the time domain and split into sections of length $\lambda_{0}$. The binned field then comprises the complex amplitudes of the fundamental spectral component of the field in each section. Figure 1 illustrates the effects of this procedure upon a sample seed. Note that this is carried out on the electric field, $E$, rather than the magnetic vector potential $A$. In order to allow easier comparison of results from the 


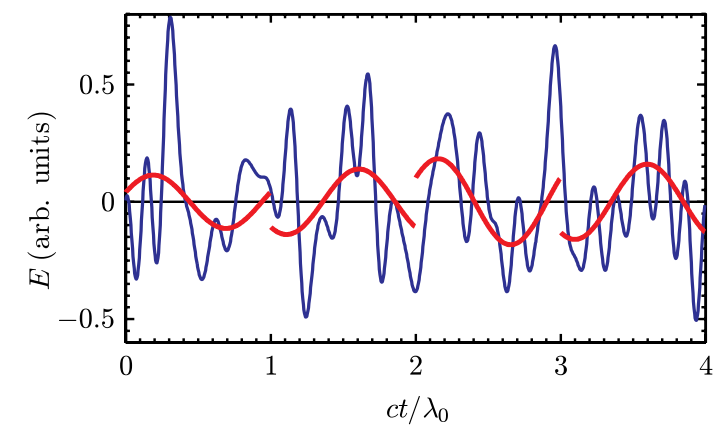

FIG. 1. A representative example of binning a broadband field (blue curve) to a grid of spacing $\lambda_{0}$ for use in a SVEA code. The discontinuous red curve sinusoids represent the phase and amplitude obtained for the fundamental frequency component within each bin.

two simulation codes, the same process is applied to AURORA radiation output.

In the frequency domain, this filtering process is equivalent to multiplication of the input field $E(\omega)$ with a filter function $W_{\lambda_{0}}\left(z_{1}, \omega\right)$ that is the product of a Gaussian filter and the Fourier transform of a top hat of width $\lambda_{0}$, centered at the bin location $z_{1}=c t-z$. On a discretized mesh the correct filter to convolve with is

$$
\begin{aligned}
W_{\lambda_{0}}\left(z_{1}, \omega\right)= & \frac{\sqrt{2 \pi}}{\omega_{0}} \exp \left[-2 \pi^{2}\left(\omega-\omega_{0}\right)^{2} / \omega_{0}^{2}\right] \\
& \times \frac{\sin \left(\pi \omega / \omega_{0}\right)}{\sin \left[\pi \omega /\left(N \omega_{0}\right)\right]} \exp \left(-i z_{1} \omega c\right),
\end{aligned}
$$

where $\lambda_{0} / N$ is the data point spacing on the grid on which the full seed is discretized.

\section{Analytical model for growth in the exponential gain regime}

For another point of reference to compare our simulation code results, we have developed an analytical model based on the linearized Maxwell-Vlasov equations (within the SVEA) similar to the development in Refs. $[25,26]$ but extended to treat fast-varying field envelopes that violate the SVEA. Here we briefly summarize our new results with fuller details given in the Appendix.

For a seed field $A(\theta, 0)$, the field at a given distance along the undulator $Z=k_{u} z$, and a given ponderomotive phase, $\theta=k_{R}(z-c t)+k_{u} z$, is expressed as

$$
A(Z, \theta)=g(Z, \theta) * A(0, \theta),
$$

where $*$ represents convolution with respect to $\theta$. Previous treatments have employed the saddle-point approximation when evaluating an expression for $g(Z, \theta)$, thereby precluding the correct modeling of fast-varying seed envelopes. In order to allow such envelopes to be modeled, in the Appendix we avoid this approximation and derive an expression based on the generalized hypergeometric function ${ }_{p} F_{q}$ [cf. Eqs. (A9) and (A11)]:

$$
\begin{aligned}
g(Z, \theta)= & \delta(Z-\theta) \\
& +8 i(Z-\theta) \theta \rho_{0}^{3} F_{2}\left[; \frac{3}{2}, 2 ; 2 i(Z-\theta)^{2} \theta \rho^{3}\right] H(Z-\theta),
\end{aligned}
$$

where $H$ is the Heaviside step function. In Sec. IVA, we compare the linearized solution from Eq. (11) for an ultrashort seed with numerical simulation results.

\section{COMPARISON OF NON-SVEA AND SVEA SIMULATION RESULTS}

Now we compare AURORA results with those from the SVEA code and, in one case, from our analytic model. We first examine the FEL response to an ultrashort input seed and then go on to examine several situations involving HHG seeds.

\section{A. Ultrashort seeds}

Our first simulation study involves an FEL seeded with a single ultrashort spike of radiation, whose high-frequency components strongly violate the SVEA. The relevant FEL parameters include resonant wavelength $\lambda_{R}=32 \mathrm{~nm}$, undulator wavelength $\lambda_{u}=20 \mathrm{~mm}$, and rms strength $a_{u}=$ 0.5 , and a monoenergetic, zero emittance electron beam with $\gamma=625$ and a current density equivalent to an FEL parameter $\rho=0.002$. The following figures use normalized, "Milanese school" units for space, time, and field measures

$\bar{z}=2 k_{u} \rho z, \quad \bar{z}_{1}=2 k_{u} \rho \frac{\bar{\beta}_{z}}{1-\bar{\beta}_{z}}(c t-z), \quad \bar{A}=\frac{k_{R} a_{u}[J J]}{4 \gamma_{R}^{2} k_{u} \rho^{2}} \frac{e}{m c} A$,

where $\bar{\beta}_{z}=\sqrt{1-1 / \gamma_{R}^{2}}$ is the mean normalized electron velocity in $z$.

Our results show that even for this case where the input seed amplitude exhibits significant variations on the scale of a resonant wavelength, there is excellent agreement between results from the SVEA and non-SVEA codes and the analytical model in the great majority of cases. Figure 2 presents a comparison between the radiation output profiles from the non-SVEA code AURORA, the PERSEOlike SVEA code mentioned previously, and the linearized analytical model at different stages of gain for a case where the initial seed had a Gaussian profile with amplitude $A_{0, \text { seed }} \sim e^{-(c t)^{2} / 2 \sigma_{\text {seed }}^{2}}$ and rms duration $\sigma_{\text {seed }}=\lambda_{R} / 8$. In terms of normalized variables, the rms width of the seed spike is $\bar{z}_{1}=\pi \times 10^{-3}$ (in other words, much shorter than the cooperation length $L_{c}$. In the SVEA code the time grid spacing for the radiation and particle quantities is $\lambda_{R} / c$ and the central wavelength $\lambda_{0}=\lambda_{R}$. The evolution of the output radiation profiles illustrate the high degree of correspondence between the results yielded by the different approaches. At $\bar{z}=10$ the simulations approach saturation 

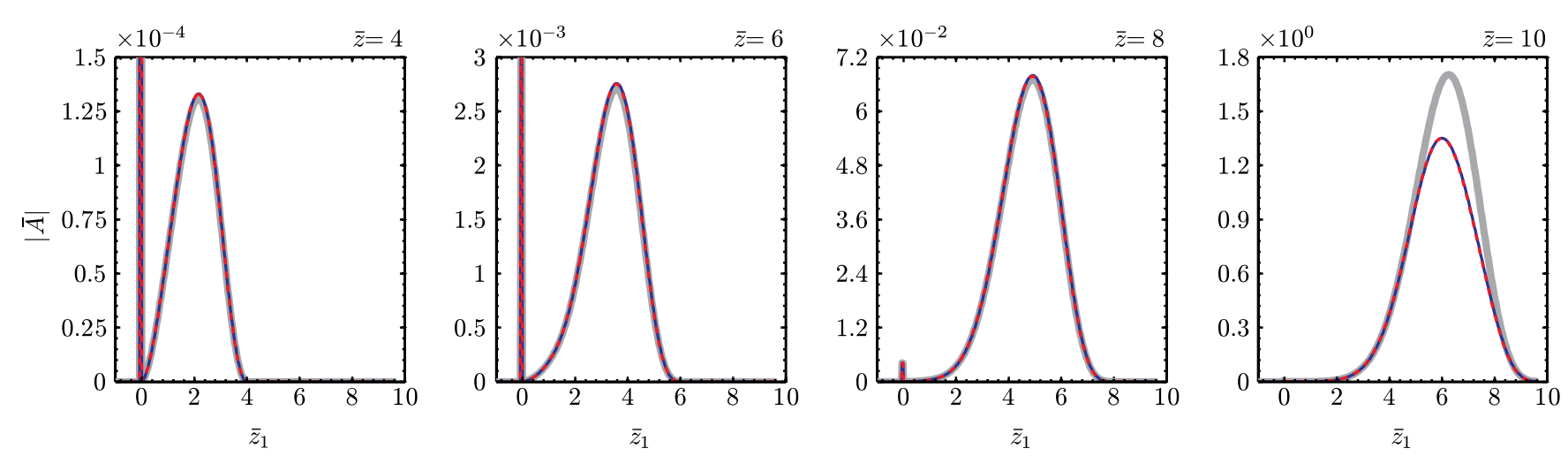

FIG. 2. Evolution at various $\bar{z}$ locations of the intensity of the radiation $A$ for a system seeded with a single ultrashort spike of rms width $\bar{z}_{1}=\pi \times 10^{-3}$ (corresponding to $\lambda_{R} / 8$ ) located at $\bar{z}_{1}=0$. The thick gray curve is from the analytical model, the solid blue from the non-SVEA code AURORA, and the dashed red curve from the conventional SVEA code. The latter almost perfectly overlaps the blue curve.

and predictably begin to deviate from the analytical model. Simulations of other ultrashort seeds with rms widths as short as $\sim \lambda_{0} / 100$ showed similar excellent agreement between results from the SVEA code initialized from a filtered seed and the non-SVEA AURORA and analytic model.

\section{B. Full HHG spectrum seeding}

In order to test further the capabilities of the simulation codes, we turn to an example that better illustrates actual HHG seeding, with an input seed spectrum comprised of odd harmonics of a hypothetical drive laser. In the time domain, this corresponds to a sequence of ultrashort spikes, two for each cycle of the driving laser. We simulate seeding with an idealized infinite sequence of such spikes by using a periodic simulation window of size $\lambda_{d}=$ $800 \mathrm{~nm}$, the drive laser wavelength. FEL resonance was set to $\lambda_{R} \approx 3.14 \mathrm{~nm}$, the 255 th harmonic of the $\lambda_{d}$. Other relevant simulation parameters include $\lambda_{u}=5 \mathrm{~mm}$, $a_{u}=0.5, \gamma=998$, and FEL parameter $\rho=5 \times 10^{-3}$. The corresponding input spectrum is shown in Fig. 3 where we also presumed that all harmonics are exactly in phase. The adopted spectrum has an exponential drop-off of intensity at the lowest harmonics and a plateau region of constant intensity per harmonic, in line with theoretical predictions and experimental observations [27]. The normalized amplitude of each of the plateau harmonics $(q=21$ to $q=281)$ is $\bar{A}_{q}=$ $2.3 \times 10^{-5}$.

Despite the large electric fields associated with the seed's long-wavelength components, their dimensionless amplitudes $a_{\text {seed }}=e A_{\text {seed }} /(m c)$ are still much less than the undulator parameter $a_{u}$, therefore we may continue to disregard their direct contribution to electron motion [cf. Eq. (9)]. Additionally, although in reality these long-wavelength components are likely to diffract significantly over the course of FEL gain (or even during preundulator transport), by neglecting diffraction effects we may consider a limiting case in terms of their potential effect on gain.

Figure 4 plots simulation results at an early stage of gain, $\bar{z}=4$, and close to FEL power saturation, $\bar{z}=14$, where $|\bar{A}| \approx 1$. Comparing the time-dependent output profiles, it is clear that the SVEA code results match those from AURORA very closely - to within the few percent levelat all stages of gain. The radiation phases exhibit a similar degree of correspondence. For purposes of comparison here, the AURORA results have also been filtered and binned according to the procedure outlined in Sec. III B. This does not, however, limit their validity, as we have verified that the filter's bandwidth covers all spectral components that experience gain.

To demonstrate the sensitivity of the SVEA code results to details of the initial Gaussian spectral filter described in Sec. III B, we have also plotted results for filters with both a wider bandwidth $\left(1.67 \omega_{0} / 2 \pi\right)$ and a narrower one $\left(0.1 \omega_{0} / 2 \pi\right)$. For the case with a broader filter, the high

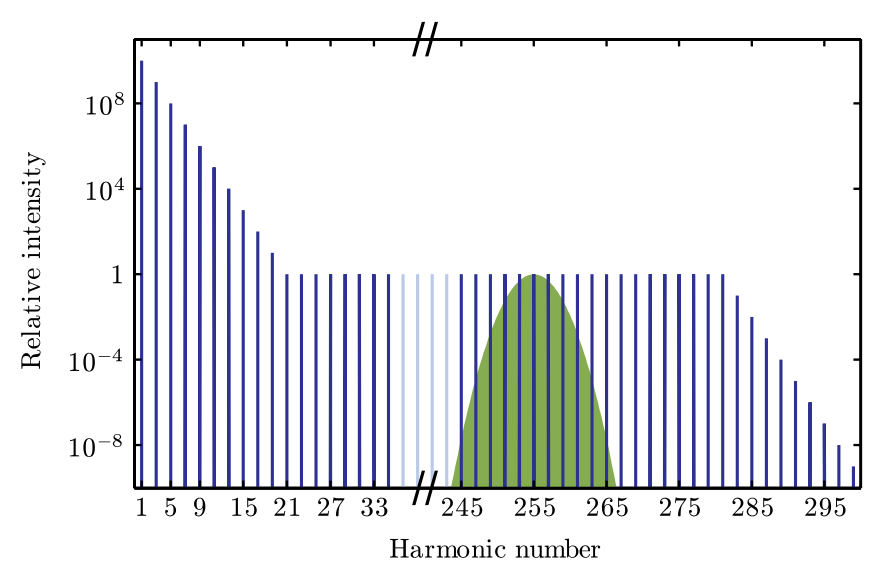

FIG. 3. Relative intensities of harmonic orders $1-35$ and 245-299 of an input HHG seed spectrum. The shaded green Gaussian around the 255th harmonic (also plotted on a logarithmic scale) represents the FEL gain curve for $\rho=5 \times 10^{-3}$. 

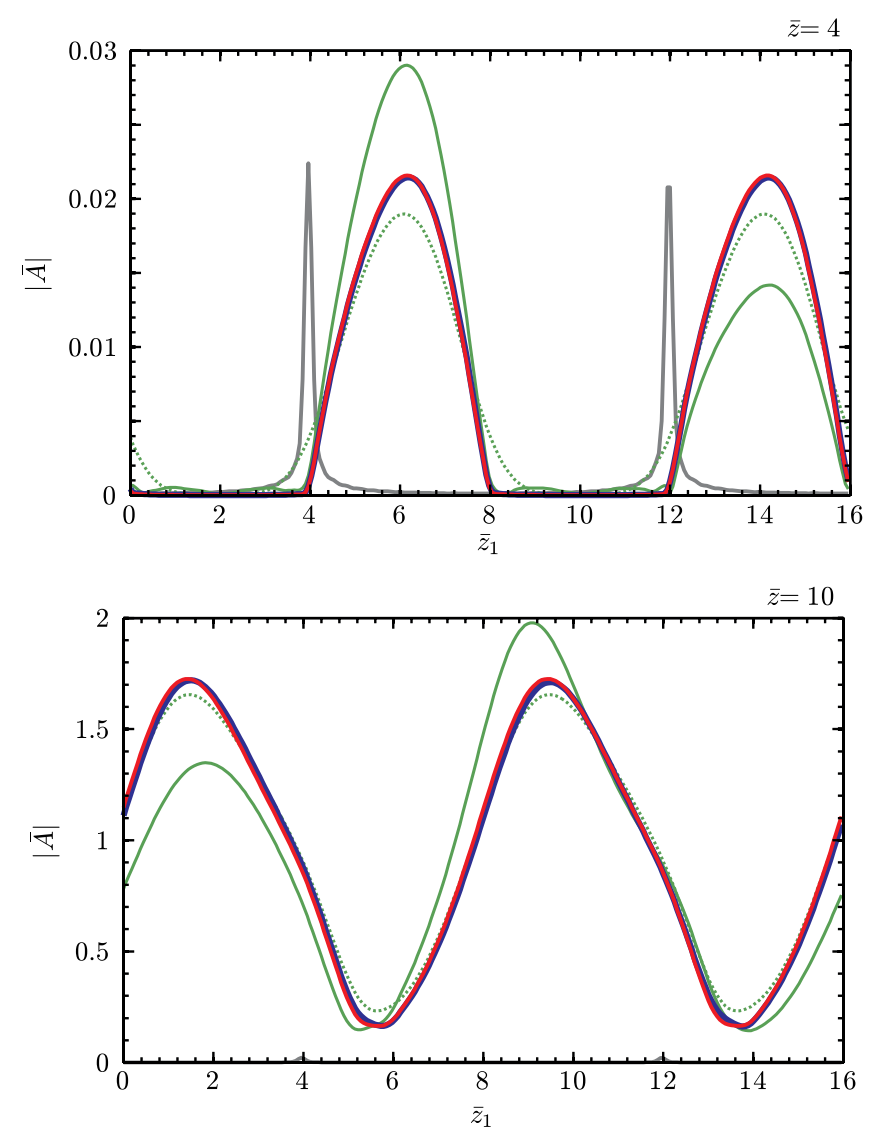

FIG. 4. Radiation output profiles versus time at $\bar{z}=4$ (top) and $\bar{z}=14$ (bottom) resulting from broadband HHG seeding (cf. seed spectrum in Fig. 3). The simulation window has a width of $\lambda_{\mathrm{d}}=$ $800 \mathrm{~nm}$ with periodic boundary conditions. The grey line represents the initial seed profile, with the characteristic two spikes per driving laser wavelength - this seed profile has been subtracted from the other results, leaving only radiation generated during FEL gain. Non-SVEA AURORA results are in blue while SVEA code results using a seed filtered with a Gaussian of rms width $\omega_{0} / 2 \pi$ are in red. The solid (dotted) green line represents SVEA simulation results where the input seed was filtered with a $1.67 \times$ broader $(10 \times$ narrower $)$ Gaussian bandpass.

intensities at low harmonics of the drive laser lead to an anomalous contribution to the seed that distorts the gain profile, as well as the total output power. In the narrowband case the seed's initially sharp temporal profile is smeared out, and thus also the profile of the output. This smearing does not, however, generally lead to large deviations in output power-especially at advanced stages of gain. This is because even the narrow filter's bandwidth still covers most of the FEL's gain bandwidth, and there is no interplay between different spectral components.

Based on the preceding analytical arguments and numerical analysis, it appears possible to accurately simulate FELs seeded with broadband radiation using conventional single-frequency codes. This is in part a manifestation of the strong mode selection that takes place during FEL gain.

\section{Evolution of HHG harmonics within FEL gain bandpass}

The full harmonic content of the seed is clearly also of importance in cases where the gain bandwidth encompasses more than one harmonic of the seeding laser. This may readily occur at high-harmonic orders, where the relative difference in frequency between adjacent harmonics becomes small. We consider again the example from the previous subsection: seeding with the 255th harmonic of an $800 \mathrm{~nm}$ driving laser at $3.14 \mathrm{~nm}$. The relative frequency separation between adjacent harmonics of that order is $\left(\omega_{255}-\omega_{257}\right) / \omega_{255}=1-255 / 257 \approx 7.8 \times 10^{-3}$, which is comparable to the gain bandwidth of a hypothetical FEL with Pierce parameter $\rho=5 \times 10^{-3}$. In this regime the presence of harmonics other than the primary seeding one may affect the gain dynamics, particularly near saturation.

We simulate this scenario in AURORA with a full seed comprising all plateau harmonic orders from 21 st to 281st at equal intensities, as illustrated in Fig. 3, and also with a filtered seed only containing the 255th harmonic. The results are presented in Fig. 5. Because of the imposed absence of initial electron bunch shot noise, for the case of the filtered seed the 255th harmonic is the only spectral component that achieves appreciable gain. The additional harmonics in the full seed undergo gain in accordance with the FEL's gain curve (inset). This difference in behavior leads to different saturation dynamics: the full seed enters saturation sooner, but takes longer to reach maximum intensity. Correspondingly, there is a period

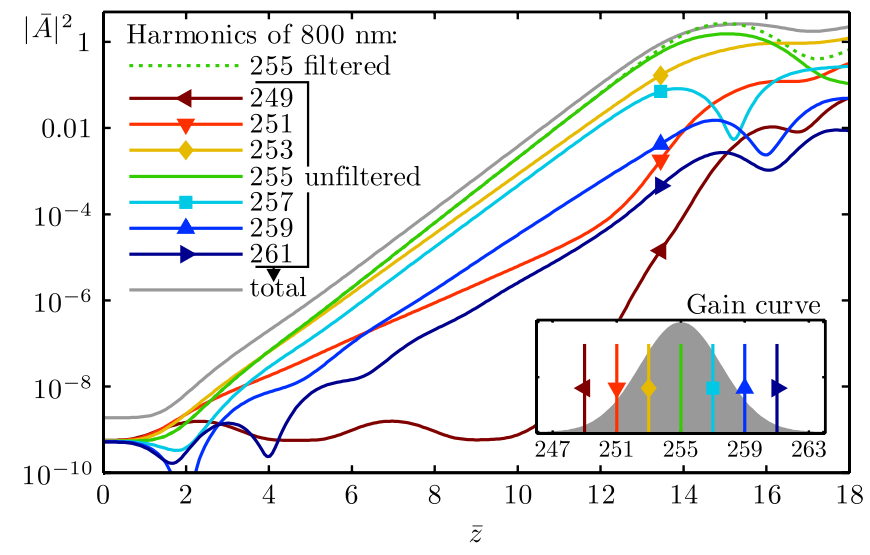

FIG. 5. Evolution of HHG seed harmonics around the FELresonant 255th harmonic of an $800 \mathrm{~nm}$ driving laser, for the cases of a full and a filtered seed. The full seed (cf. Fig. 3) contains several harmonics that fall within the gain bandwidth $\left(\rho=5 \times 10^{-3}\right)$, whereas the filtered seed case contains only the 255th harmonic. Spectral components of FEL output resulting from the full seed are represented by solid lines, where the gray line is total normalized field due to harmonics 249 through 261; the output from the filtered seed is represented by the dotted green line. Inset: harmonic content of the full seed (vertical lines), set against the FEL gain bandwidth (gray). 
around $\bar{z} \approx 15$ where the filtered seed actually yields a marginally greater intensity than the full seed. The "tapered saturation" exhibited by the full seed is attributable to the fact that different modes within the gain curve saturate at different times, and in doing so inhibit the saturation of other modes. Thus, for example, in the case of the full seed the 255th harmonic can never reach the same saturation intensity as in the case of the filtered seed.

As demonstrated in the previous subsection, even this case-where seed harmonics other than the primary seeding harmonic have an effect on FEL gain - can in fact be simulated in a conventional SVEA-compliant FEL code, as long as the seed field is appropriately binned. This relates to the arguments of Sec. II, where we showed that dropping the SVEA is not necessary even when the FEL is seeded with radiation that violates the approximation. Indeed, the SVEA only needs to be dropped when the radiation emitted by the FEL has a fast-varying profile-the most striking regime where this is the case is when the Pierce parameter approaches unity, i.e., we no longer have $\rho \ll 1$. This corresponds to very broadband emission, as well as significant amplification on the scale of a single undulator period. This regime is currently under investigation, and initial studies suggest that working outside the SVEA is indeed necessary in order to accurately simulate it.

\section{CONCLUSION}

We have considered seeding of free-electron lasers with radiation pulses containing frequency components that strongly violate the approximations that lead to the slowly varying envelope approximation (SVEA). We have considered both ultrashort seeds whose duration is comparable to the period of their central wavelength and also HHG radiation that consists of a series of ultrashort spikes, the amplitude of which may vary on the scale of the resonant FEL wavelength.

We have shown that, as a result of the way seed radiation is represented and propagated in 1D FEL codes, SVEA code results appear to remain valid even when the seed violates that approximation. We have confirmed this through FEL simulations carried out both with and without the SVEA, the latter carried out using the 1D multifrequency non-undulator-period-averaged code AURORA, which was in part developed for this purpose. Additional comparisons were carried out against an analytical linearized model, again based on the SVEA. Provided that the full broadband seed was filtered and binned appropriately prior to being used as an input to an SVEA-based code, the three methods yield essentially identical results. This appears true for both ultrashort and or wideband HHG seeds. We also discuss the case where the gain bandwidth is greater than the harmonic spacing in an HHG seed. Here there is minor impairment of output power in the primary seeded harmonic.
These results consider a single spatial dimension. While transverse effects such as radiation diffraction will affect the FEL's overall characteristics, it is unlikely there would be an interplay between these effects and the temporal and spectral characteristics of FEL gain, such that wideband effects are more important in 3D than 1D. In fact, the much greater amount of diffraction at long wavelengths suggests that there will be an automatic high passband filtering of HHG seeds, even further limiting non-SVEA effects.

\section{ACKNOWLEDGMENTS}

The authors would like to acknowledge stimulating discussions with E. Esarey, W. Leemans, and B. McNeil. S. B. acknowledges E. Bajlekova, T. Robinson, and K. O'Keeffe. We are grateful for financial support from the Leverhulme Trust (Grant No. F/08 776/G); the Engineering and Physical Sciences Research Council (Grant No. EP/ H011145/1); and the Director, Office of Science, of the U.S. Department of Energy under Contract No. DE-AC02$05 \mathrm{CH} 11231$.

\section{APPENDIX: LINEARIZED MAXWELL-VLASOV EQUATIONS}

Following a similar approach as in Refs. [25,26], we derive the Green's function for amplification of a seed, Eq. (11). The electron bunch is characterized by the distribution function $f(Z, \theta, \eta)$, where $Z=k_{u} z, \theta=\left(k+k_{u}\right) z^{-}$ $k c t$, and $\eta=\left(\gamma-\gamma_{0}\right) / \gamma_{0}$ are dimensionless variables. The distribution's evolution is governed by the Vlasov equation,

$$
d f=\left(\partial_{Z}+\dot{\theta} \partial_{\theta}+\dot{\eta} \partial_{\eta}\right) f=0 .
$$

The dotted terms are derivatives of the phase space variables with respect to $Z$, correspondingly

$$
\dot{\theta}=2 \eta, \quad \dot{\eta}=-\kappa_{B} a e^{i \theta},
$$

where $a \equiv e A / m c$ is the normalized field envelope. The Maxwell equation governing the latter is

$$
\left(\partial_{Z}+\partial_{\theta}\right) a=\kappa_{M} e^{-i \theta} \int d \eta f .
$$

The two dimensionless constants are

$$
\kappa_{M}=\frac{n_{e} e^{2} a_{u}[J J]}{4 c^{2} \gamma_{0} k k_{u} m \varepsilon_{0}}, \quad \kappa_{B}=\frac{k a_{u}[J J]}{2 k_{u} \gamma_{0}^{2}},
$$

where $n_{e}$ is the peak electron density and all other parameters are as defined in Sec. II. We note that $\kappa_{M} \kappa_{B}=4 \rho^{3}$.

We rewrite the distribution function as $f=f_{0}+f_{1}$, where $f_{0}$ is the initial distribution and $f_{1}$ accounts for FEL-induced modulation. The shot-noise contribution is not considered here. Linearizing Eq. (A1) results in

$$
\left(\partial_{Z}+\dot{\theta} \partial_{\theta}\right) f_{0}=0
$$

and 


$$
\left(\partial_{Z}+\dot{\theta} \partial_{\theta}\right) f_{1}=\kappa_{B} a e^{i \theta} \partial_{\eta} f_{0},
$$

which are valid during the start-up and exponential gain regimes, where $a \ll 1$. Rearranging Eq. (A4), we obtain

$$
\left[\partial_{Z}+\dot{\theta}\left(i+\partial_{\theta}\right)\right]\left(e^{-i \theta} f_{1}\right)=\kappa_{B} a \partial_{\eta} f_{0} .
$$

We solve this in the Fourier domain, $\mathcal{F}[f(\theta)]=F(\nu)$ :

$$
\mathcal{F}\left[e^{-i \theta} f_{1}\right]=\int_{0}^{Z} d Z^{\prime} e^{-i \dot{\theta}(1+\nu)\left(Z-Z^{\prime}\right)} \kappa_{B} \mathcal{F}\left[a\left(Z^{\prime}\right) \partial_{\eta} f_{0}\left(Z^{\prime}\right)\right] .
$$

Carrying out the inverse transform integrating over $\nu$ we obtain

$$
e^{-i \theta} f_{1}=\kappa_{B} \int_{0}^{Z} d Z^{\prime} e^{i \dot{\theta}\left(Z^{\prime}-Z\right)} a\left(Z^{\prime}, \theta^{\prime}\right) \partial_{\eta} f_{0}\left(Z^{\prime}, \theta^{\prime}, \eta\right)
$$

with $\theta^{\prime}=\theta-\dot{\theta}\left(Z-Z^{\prime}\right)$. Finally, from Eq. (A3) $f_{0}(Z, \theta, \eta)=f_{0}(\theta-\dot{\theta} Z, \eta)$, and thus also $f_{0}\left(Z^{\prime}, \theta^{\prime}, \eta\right)=$ $f_{0}(\theta-\dot{\theta} Z, \eta)$.

Substituting the above into Eq. (A2) yields

$$
\left(\partial_{Z}+\partial_{\theta}\right) a=\kappa_{M} \int d \eta e^{-i \theta} f_{1}=4 \rho^{3} \int_{0}^{Z} d Z^{\prime} e^{i \dot{\theta}\left(Z^{\prime}-Z\right)} a\left(Z^{\prime}, \theta^{\prime}\right) \int d \eta \partial_{\eta} f_{0}(\theta-\dot{\theta} Z, \eta) .
$$

This is amenable to solution via a Laplace transform, $\mathcal{L}[a(Z)]=A(s)$,

$$
\left(s+\partial_{\theta}\right) A(s, \theta)-a(Z=0, \theta)=4 \rho^{3} \int_{0}^{\infty} d Z e^{-s Z} \int_{0}^{Z} d Z^{\prime} e^{i \dot{\theta}\left(Z^{\prime}-Z\right)} a\left(Z^{\prime}, \theta^{\prime}\right) \int d \eta \partial_{\eta} f_{0}(\theta-\dot{\theta} Z, \eta) .
$$

Since $a\left(\theta^{\prime}\right) f_{0}(\theta-\dot{\theta} Z)$ contains higher-order terms, under linearization it becomes $a(\theta) f_{0}(\theta)$ :

$$
\begin{aligned}
\left(s+\partial_{\theta}\right) A(s, \theta)-a(Z=0, \theta) & =4 \rho^{3} \int d \eta \partial_{\eta} f_{0}(\theta, \eta) \int_{0}^{\infty} d Z e^{-(s+i \dot{\theta}) Z} \int_{0}^{Z} d Z^{\prime} e^{i \dot{\theta} Z^{\prime}} a\left(Z^{\prime}, \theta\right) \\
& =4 \rho^{3} \int d \eta \partial_{\eta} f_{0}(\theta, \eta) \int_{0}^{\infty} d Z^{\prime} e^{-s Z^{\prime}} \frac{a\left(Z^{\prime}, \theta\right)}{s+i \dot{\theta}}=4 \rho^{3} \int d \eta \partial_{\eta} f_{0}(\theta, \eta) \frac{A(s, \theta)}{s+i \dot{\theta}} .
\end{aligned}
$$

Rearranging and integrating by parts, we obtain

$$
\begin{aligned}
\left(s+\partial_{\theta}\right) A(s, \theta)= & a_{0}(\theta)+i(2 \rho)^{3} A(s, \theta) \\
& \times \int d \eta \frac{f_{0}(\theta, \eta)}{(s+2 i \eta)^{2}},
\end{aligned}
$$

where the initial (seed) field is represented by $a_{0}(\theta)=$ $a(Z=0, \theta)$. Finally, we assume an initially cold, longitudinally uniform electron beam, $f_{0}(\theta, \eta)=\delta(\eta)$ :

$$
\left[s+\partial_{\theta}-\frac{i(2 \rho)^{3}}{s^{2}}\right] A(s, \theta)=a_{0}(\theta) .
$$

The solution to this equation is

$$
A(s, \theta)=\int_{-\infty}^{\theta} d \theta^{\prime} \exp \left[\left(-s+\frac{i(2 \rho)^{3}}{s^{2}}\right)\left(\theta-\theta^{\prime}\right)\right] a_{0}\left(\theta^{\prime}\right),
$$

and carrying out the inverse Laplace transform,

$$
a(Z, \theta)=\mathcal{L}^{-1}\left\{\exp \left[-s \theta+\frac{i(2 \rho)^{3}}{s^{2}} \theta\right]\right\} * a_{0}(\theta)
$$

where $*$ represents convolution with respect to $\theta$.

Defining $\chi \equiv i(2 \rho)^{3} \theta$, we go on to evaluate the inverse transform in Eq. (A7). From the power series expansion

$$
\frac{1}{s} \exp \left(\frac{\chi}{s^{2}}\right)=\sum_{n=0}^{\infty} \frac{\chi^{n}}{s^{2 n+1} n !},
$$

applying the relation $\mathcal{L}^{-1}\left\{\frac{1}{s^{n+1}}\right\}=\frac{Z^{n}}{n !}$, we obtain the inverse Laplace transform

$$
\mathcal{L}^{-1}\left\{\frac{1}{s} \exp \left(\frac{\chi}{s^{2}}\right)\right\}=\sum_{n=0}^{\infty} \frac{\chi^{n} Z^{2 n}}{n !(2 n) !} \equiv G(Z, \theta) .
$$

Using

$$
\mathcal{L}^{-1}\{s F(s)\}=f^{\prime}(Z)+f(0) \delta(Z)
$$

and

$$
\mathcal{L}^{-1}\{\exp (-s \theta) F(s)\}=f(Z-\theta) H(Z-\theta),
$$

where $\mathcal{L}^{-1}\{F(s)\}=f(Z)$, and $H$ is the Heaviside step function, we obtain

$$
\begin{aligned}
\mathcal{L}^{-1} & \left\{\exp (-s \theta) \exp \left(\chi / s^{2}\right)\right\} \\
= & \frac{\partial G}{\partial Z}(Z-\theta, \theta) H(Z-\theta)+\delta(Z-\theta) .
\end{aligned}
$$

The solution for $\partial G / \partial Z$ can be expressed in terms of the generalized hypergeometric function, ${ }_{p} F_{q}$,

$$
\begin{aligned}
\frac{\partial G}{\partial Z}(Z-\theta, \theta) & =\sum_{n=1}^{\infty} \frac{\left[i(2 \rho)^{3} \theta\right]^{n}(Z-\theta)^{2 n-1}}{n !(2 n-1) !} \\
& ={ }_{0} F_{2}\left[; 1,1 / 2 ; i\left(2 \rho^{3}\right) \theta Z^{2}\right],
\end{aligned}
$$

where

$$
p F_{q}\left(a_{1}, \ldots, a_{p} ; b_{1}, \ldots, b_{q} ; z\right)=\sum_{k=0}^{\infty} \frac{\left(a_{1}\right)_{k}\left(a_{2}\right)_{k} \cdots\left(a_{p}\right)_{k}}{\left(b_{1}\right)_{k}\left(b_{2}\right)_{k} \cdots\left(b_{q}\right)_{k}} \frac{z^{k}}{k !}
$$

with $(a)_{k} \equiv a(a+1) \cdots(a+k-1) ;(a)_{0} \equiv 1$. 
For values of $Z \lesssim \pi / \rho$ (beyond which nonlinear effects manifest) and $\theta \in(0, Z)$, the series in Eq. (A9) is accurately approximated by its first four terms:

$$
\begin{aligned}
\frac{\partial G}{\partial Z}(Z-\theta, \theta) \approx & 8 i(Z-\theta) \theta \rho^{3}-\frac{16}{3}(Z-\theta)^{3} \theta^{2} \rho^{6} \\
& -\frac{32}{45} i(Z-\theta)^{5} \theta^{3} \rho^{9}+\frac{32}{945}(Z-\theta)^{7} \theta^{4} \rho^{12}
\end{aligned}
$$

[1] S. Doniach, J. Synchrotron Radiat. 3, 260 (1996).

[2] R. Neutze, R. Wouts, D. van der Spoel, E. Weckert, and J. Hajdu, Nature (London) 406, 752 (2000).

[3] P. Emma et al., Nat. Photon. 4, 641 (2010).

[4] M. Altarelli et al., The European X-Ray Free-Electron Laser Technical Design Report (DESY, Hamburg, Germany, 2007).

[5] T. Shintake et al., Phys. Rev. ST Accel. Beams 12, 070701 (2009).

[6] R. Bonifacio, C. Pellegrini, and L. M. Narducci, Opt. Commun. 50, 373 (1984).

[7] R. Bonifacio and F. Casagrande, Nucl. Instrum. Methods Phys. Res., Sect. A 239, 36 (1985).

[8] P. B. Corkum and F. Krausz, Nature Phys. 3, 381 (2007).

[9] G. Lambert et al., Nature Phys. 4, 296 (2008).

[10] T. Hara et al., in Proceedings of the 32nd International Free Electron Laser Conference, Malmö, Sweden (2010).

[11] L. Giannessi, M. Quattromini, P. Musumeci, M. Nisoli, G. Sansone, S. Stagira, and S. de Silvestri, in Proceedings of the 28th International Free Electron Laser Conference (BESSY, Berlin, Germany, 2006), p. 248.
[12] B. W. J. McNeil, D. Dunning, N. R. Thompson, and B. Sheehy, in Proceedings of the 28th International Free Electron Laser Conference (Ref. [11]), p. 234.

[13] J. Wu, P. R. Bolton, J. B. Murphy, and X. Zhong, Appl. Phys. Lett. 90, 021109 (2007).

[14] G. Sansone, E. Benedetti, F. Calegari, C. Vozzi, L. Avaldi, R. Flammini, L. Poletto, P. Villoresi, C. Altucci, R. Velotta, S. Stagira, S. De Silvestri, and M. Nisoli, Science 314, 443 (2006).

[15] L. Giannessi, in Proceedings of the 28th International Free Electron Laser Conference (Ref. [11]), p. 91.

[16] C. Maroli, Opt. Commun. 208, 155 (2002).

[17] C. K. Birdsall and A. B. Langdon, Plasma Physics Via Computer Simulation (McGraw Hill Book Co., New York, 1985).

[18] B. W. J. McNeil and G. R. M. Robb, Phys. Rev. E 65, 046503 (2002).

[19] N. Piovella, Phys. Plasmas 6, 3358 (1999).

[20] S. I. Bajlekov, R. Bartolini, and S.M. Hooker, in Proceedings of the 31st International Free Electron Laser Conference, Liverpool, UK (STFC Daresbury Laboratory, Warrington, 2009), p. 119.

[21] L. T. Campbell, R. Martin, and B.W. J. McNeil, in Proceedings of the 31st International Free Electron Laser Conference, Liverpool, UK (Ref. [20]), p. 115.

[22] C. Penman and B.W. J. McNeil, Opt. Commun. 90, 82 (1992).

[23] W. M. Fawley, Phys. Rev. ST Accel. Beams 5, 070701 (2002).

[24] B. W. J. McNeil, M. W. Poole, and G. R. M. Robb, Phys. Rev. ST Accel. Beams 6, 070701 (2003).

[25] S. Krinsky and L. H. Yu, Phys. Rev. A 35, 3406 (1987).

[26] S. Krinsky and Z. Huang, Phys. Rev. ST Accel. Beams 6, 050702 (2003).

[27] J. Chen, S.-I. Chu, and J. Liu, J. Phys. B 39, 4747 (2006). 